\title{
Pion transition form factor in the Regge approach and incomplete vector-meson dominance *
}

\author{
Enrique Ruiz Arriola ${ }^{1,0}$ 田 and Wojciech Broniowski ${ }^{2}, 3$, 团 \\ ${ }^{1}$ Departamento de Física Atómica, Molecular y Nuclear, \\ Universidad de Granada, E-18071 Granada, Spain \\ ${ }^{2}$ The H. Niewodniczański Institute of Nuclear Physics, PL-31342 Kraków, Poland \\ ${ }^{3}$ Institute of Physics, Jan Kochanowski University, PL-25406 Kielce, Poland
}

(Dated: 5 April 2010)

\begin{abstract}
The concept of incomplete vector-meson dominance and Regge models is applied to the transition form factor of the pion. First, we argue that variants of the chiral quark model fulfilling the chiral anomaly may violate the Terazawa-West unitarity bounds, as these bounds are based on unverified assumptions for the real parts of the amplitudes, precluding a possible presence of polynomial terms. A direct consequence is that the transition form factor need not necessarily vanish at large values of the photon virtuality. Moreover, in the range of the BaBar experiment, the Terazawa-West bound is an order of magnitude above the data, thus is of formal rather than practical interest. Then we demonstrate how the experimental data may be properly explained with incomplete vectormeson dominance in a simple model with one state, as well as in more sophisticated Regge models. Generalizations of the simple Regge model along the lines of Dominguez result in a proper description of the data, where one may adjust the parameters in such a way that the Terazawa-West bound is satisfied or violated. We also impose the experimental constraint from the $Z \rightarrow \pi_{0} \gamma$ decay. Finally, we point out that the photon momentum asymmetry parameter may noticeably influence the precision analysis.
\end{abstract}

PACS numbers: 12.38.Lg, 11.30, 12.38.-t

Keywords: Pion transition form factor, large- $N_{c}$ Regge models, chiral anomaly, incomplete vector-meson dominance, chiral quark models, perturbative QCD

\section{INTRODUCTION}

The pion transition form factor, $F_{\pi^{0} \gamma \gamma^{*}}$, measured in the $e^{+} e^{-} \rightarrow \pi^{0} \gamma$ annihilation, has been a particularly interesting object of study since its value at the origin is fixed by the chiral anomaly [1, 2] while the behavior at very high Euclidean momenta has been predicted via perturbative quantum chromodynamics (pQCD), apparently holding at sufficiently high but unspecified scales [3, 4]. However, objections have been raised [5] and reiterated [6] against the applicability of pQCD to exclusive processes. The recent measurement of $F_{\pi^{0} \gamma \gamma^{*}}$ by the BaBar Collaboration [7], where the pion transition form factor goes visibly above the conventional pQCD prediction at scales $Q^{2}>15 \mathrm{GeV}^{2}$, raised serious doubts concerning the theoretical understanding of the exclusive processes, also in the asymptotic region. In the pQCD approach based on factorization, one uses the light-cone Feynman rules and the ERBL evolution of the pion distribution amplitude (PDA) 3, 4, , 8- 16], which results asymptotically in the leading-twist Brodsky-Lepage

\footnotetext{
* Supported by Polish Ministry of Science and Higher Education grant N202 249235, Spanish DGI and FEDER funds with grant FIS2008-01143/FIS, Junta de Andalucía grant FQM225-05, and EU Integrated Infrastructure Initiative Hadron Physics Project contract RII3-CT-2004-506078.

${ }^{\dagger}$ Electronic address: earriola@ugr.es

${ }_{\ddagger}^{\ddagger}$ Electronic address: Wojciech.Broniowski@ifj.edu.pl
}

term $F_{\pi^{0} \gamma \gamma^{*}} \sim 2 f_{\pi} / Q^{2}$, in vivid contradiction to the recent BaBar [7] data.

Several ideas have been proposed to circumvent the problem. Radyushkin [17] pointed out that the presence of the possible end-point singularities in the PDA (as found by the authors in the chiral quark models at the low-energy quark-model scale [18]), together with essentially switched-off evolution and regulated quark propagators, is capable of reproducing the data in the available $Q^{2}$ domain. Similar conclusions were reached by Polyakov [19]. In this approach asymptotically $F_{\pi^{0} \gamma \gamma^{*}} \sim$ $\log \left(Q^{2} / \mu^{2}\right) / Q^{2}$, with the $\log$ in the numerator indicating the breaking of factorization. We note that the same asymptotics follows in the Spectral Quark Model (SQM), see Eq. (14.1) of Ref. 20]. In fact, serious concerns have been spoken out on the validity of factorization 21] in the considered process. Dorokhov [22 24] proposed the use of the fixed-mass constituent quark model to evaluate the triangle diagram of Fig. 1, which is capable of reproducing the BaBar data, however the needed value of the constituent quark mass is uncomfortably low, $M \sim 135 \mathrm{MeV}$. In this model the asymptotics has the form $\sim\left[\log \left(Q^{2} / \mu^{2}\right)\right]^{2} / Q^{2}$. Possible need of the highertwist terms has been invoked in Ref. [25]. The calculation of Kotko and Praszalowicz [26] in the non-local chiral quark model inspired by the instanton-liquid model of QCD produced the result in agreement with the data at lower values of $Q^{2}$. The influence of non-perturbative gluonic components of the pion stemming from instantons has been considered in Ref. 27]. Mikhailov and Stefanis [16] showed that the significant experimental growth 
of the transition form factor between 10 and $40 \mathrm{GeV}^{2}$ cannot be explained in terms of higher-order pQCD corrections at the NNLO level. Finally, in a very recent paper 28] Dorokhov reiterates the findings of Ref. 22. in the non-local model, where the asymptotic behavior is $\sim \log \left(Q^{2} / \mu^{2}\right) / Q^{2}$. The non-local model of Ref. [28] also requires a very low constituent quark mass in order to reproduce the data in the whole $Q^{2}$ range.

Given the fact that the recent BaBar data [7] predate the conventional pQCD expectations at such high virtualities as $15-30 \mathrm{GeV}^{2}$, it seems adequate to question any implicit assumptions in theoretical analyses. In this paper we analyze critically some of the assumptions underlying certain high-energy theorems which, if holding, would forbid the simple pre-BaBar fits based on the incomplete vector-meson dominance (IVMD) 29] (see also Ref. [30] where this result is upgraded with tiny consequences for to the muon $g-2$ ). The theorems in question, the Terazawa-West (TW) bounds [31, 32], were recalled by Dorokhov in Ref. 24].

The TW bounds [31, 32] (for a review see, e.g., [33]) are central to our discussion; they are reviewed in Sec. III] Their derivation uses the Schwarz inequality to predict that for physical momenta $\operatorname{Im} F_{\pi^{0} \gamma \gamma^{*}}=\mathcal{O}\left(1 / \sqrt{q^{2}}\right)$ (the first bound). If one further assumes that there are no polynomial contributions to the real part of the form factor, one may conclude with the help of a dispersion relation that $F_{\pi^{0} \gamma \gamma^{*}}=\mathcal{O}(1 / Q)$ for space-like momenta $Q$ (the second bound). The assumption concerning the real part is clearly spelled out in the original works and, to our knowledge, has never been credibly questioned. We should remind the reader that this was a pre-QCD bound, so we may test the validity of the assumption by analyzing a particular model where both the anomaly is satisfied and the second Terazawa-West bound is violated. Actually, a reanalysis of the issue using the quark model and corresponding triangle graphs was presented in Ref. [34] as an example case where the expected bound behavior holds. Here we will consider another variant of the quark model which in fact does not fulfill the assumptions, hence leads to a violation of the second bound. The key point is to realize that the anomaly fixes the value of the form factor at the origin, while the finite $Q$ behavior can be determined regardless of the value at $Q=0$. This construction requires a subtraction constant which actually implies that at large $Q$ the pion transition form factor does not go to zero. These arguments suggest that pQCD might in fact be computing just the subtracted form factor. Clearly, the issue cannot be settled unless one could go smoothly over all the available energy range. Of course, quark models are not QCD itself, and it is quite possible that our considerations do not apply directly to the QCD analyses. However, the present paper unveils a warning that from purely field-theoretic reasons an asymptotically non-vanishing pion transition form factor cannot be rejected.

With this finding in mind we analyze in detail the predictions of IVMD and the Regge models for $F_{\pi^{0} \gamma \gamma^{*}}$.

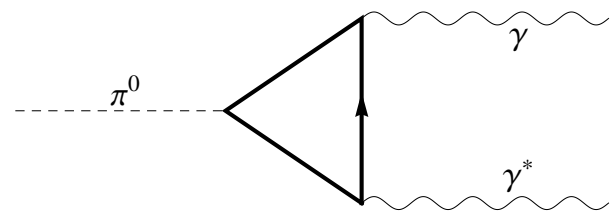

FIG. 1: The triangle diagram used to evaluate the pion transition form factor in quark models, with one real and one virtual photon (the crossed diagram not shown).

Regge models with (infinitely many) tree-level meson and glueball exchanges are a realization of the large- $N_{c}$ limit [35] which, unlike the quark models, incorporates confinement. In particular, these models where insightful in analyzing condensate issues [36-44], form factors [45, 46], or the nature of scalar states [47].

On purely phenomenological grounds, we will show that the data for $F_{\pi^{0} \gamma \gamma^{*}}$ can be fitted very well in a simple vector-meson exchange model with IVMD. In more elaborate variants of the Regge approach the data may be reproduced (with different choices of the model parameters) almost equally well with or without the fulfillment of the TW bounds.

There has also been an important interest in the pion transition form factor after the proposal of determining the rare $Z \rightarrow \pi^{0} \gamma$ decay [48], which probes the timelike value $q^{2}=M_{Z}^{2}$. The experimental bound given by the Particle Data Group [49] implies a large suppression as compared to the $q^{2}=0$ anomaly point, but not necessarily as much as predicted by many authors, where $\Gamma\left(Z \rightarrow \pi^{0} \gamma\right) \sim 10^{-11} \mathrm{GeV}$ (see, e.g., [50]).

The paper is organized as follows. As a preparatory material, in Sec. II we provide some basic kinematics, in Sec. III we review the TW bounds, and in Sec. IV recall the bound from the $Z \rightarrow \pi^{0} \gamma$ decay. In Sec. V we use several variants of the quark model to inquire on the validity of the TW bounds. As we will show, these models are not capable of describing the data with realistic model parameters, but pose a warning on the second TW bound. We use this insight in Sec. VI to propose a more realistic Vector Meson Dominance description of the data. The analysis is complemented in Sec. VII with the inclusion of the large $N_{c}$ motivated infinite tower of radial Regge like excitations of the $\rho$-meson. The important role of photon asymmetry is discussed briefly in Sec.VIII Finally, in Sec. IX] we list our main conclusions.

\section{THE KINEMATICS}

With the outgoing momenta and polarizations of the photons denoted as $q_{1}, e_{1}$ and $q_{2}, e_{2}$ (see Fig. (1), one finds the amplitude

$$
\Gamma_{\pi^{0} \gamma^{*} \gamma^{*}}^{\mu \nu}\left(q_{1}, q_{2}\right)=\epsilon_{\mu \nu \alpha \beta} e_{1}^{\mu} e_{2}^{\nu} q_{1}^{\alpha} q_{2}^{\beta} F_{\pi \gamma^{*} \gamma^{*}}\left(Q^{2}, A\right),
$$

where the pion transition form factor $F_{\pi \gamma^{*} \gamma^{*}}$ depends on the total virtuality, $Q^{2}$, and the photon momentum 
asymmetry parameter, $A$,

$$
Q^{2}=-\left(q_{1}^{2}+q_{2}^{2}\right), \quad A=\frac{q_{1}^{2}-q_{2}^{2}}{q_{1}^{2}+q_{2}^{2}},-1 \leq A \leq 1 .
$$

Equivalently, $q_{1}^{2}=-\frac{(1+A)}{2} Q^{2}, q_{2}^{2}=-\frac{(1-A)}{2} Q^{2}$. For large virtualities, assuming factorization, one finds the standard twist decomposition of the pion transition form factor [8],

$$
F_{\pi^{0} \gamma^{*} \gamma^{*}}\left(Q^{2}, A\right)=J^{(2)}(A) \frac{1}{Q^{2}}+\ldots
$$

with

$$
J^{(2)}(A)=\frac{4 f}{N_{c}} \int_{0}^{1} d x \frac{\varphi_{\pi}^{(2)}(x)}{1-(2 x-1)^{2} A^{2}},
$$

involving the pion parton distribution amplitude PDA, $\varphi(x)$.

Taking the asymptotic form $\varphi(x)=6 x(1-x)$ in Eq. (4) gives the Brodsky-Lepage asymptotic result [8]

$$
J^{(2)}(A=1)=\frac{6}{N_{c} f} .
$$

An analysis of the lowest Gegenbauer moments $a_{2}$ and $a_{4}$ of $\varphi(x)$ has been carried out in Ref. [51] and [13 15, 52]. Higher twists have been analyzed in the framework of the light-cone sum rules 53. A direct measurement of the DA has been presented by the E791 collaboration [54]. For a concise review on all these developments see, e.g., Ref. 55] and references therein.

Although theoretically one may take the maximally asymmetric case of $A=1$, in the experiment this situation is not possible to achieve. For instance, in the BaBar kinematic setup $-q_{1}^{2}<0.6 \mathrm{GeV}^{2}$ and $-q_{2}^{2}>3 \mathrm{GeV}^{2}$, suggesting $A \sim 0.9-0.97$. As advanced in Ref. [56], we note that the model results presented in the following sections become quite sensitive to the precise value of $A$ at large $Q^{2}$, hence in precision analyses the effects of kinematic cuts should be incorporated.

At this point, before undertaking further elaborations, an important qualification is in order. Eq. (4) provides a gauge-invariant high-energy-motivated definition of the pion PDA, which formally determines a non-perturbative matrix element. A purely low-energy definition of the PDA involves link operators between the quark fields at different space-time points and depends on the renormalization scale. A non-trivial issue has to do with the equivalence of both definitions and the scale at which this identification makes sense. We refer to previous works [18, 20, 45, 57] for a detailed discussion on this delicate and non-trivial issue. An outstanding outcome was that in several models

$$
\varphi(x)=1
$$

at the model low-energy scale; the end-point behavior would be bended after the QCD evolution to higher scales (see Eq. (5.26) of Ref. [58] for the detailed asymptotic form of the PDA near the end-points after evolution). While the identification of the PDA through Eq. (41) indeed requires taking asymptotically large momenta, it does not necessarily follow that the asymptotic expression itself holds at the so far measured momenta.

\section{TERAZAWA-WEST BOUNDS}

Terazawa [31] and West 32] (for a review see, e.g., 33]) derived unitarity bounds for the pion transition form factor. The derivation considers the photon propagator, the charge form factor of the pion, and the transition form factor. The Schwarz inequality is used to show that

$$
\operatorname{Im} F_{\pi^{0} \gamma \gamma^{*}}\left(q^{2}\right)=\mathcal{O}\left(1 / \sqrt{q^{2}}\right)
$$

for time-like momenta, $q^{2}>4 m_{\pi}^{2}$, which we term the first $T W$ bound. Under a further important assumption that there are no polynomial contributions to the real part of the form factor, one may also conclude that $\left|F_{\pi^{0} \gamma \gamma^{*}}\left(q^{2}\right)\right|=\mathcal{O}\left(1 / \sqrt{q^{2}}\right)$. Then the dispersion relation (see Sec. 14 of Ref. [33]) yields that

$$
\left|F_{\pi^{0} \gamma \gamma^{*}}\left(Q^{2}\right)\right|=\mathcal{O}(1 / Q)
$$

for large space-like momenta $Q$, which we call the second $T W$ bound.

The assumption of the absence of the polynomial terms (in particular, a constant) from the real part of the pion transition form factor is equivalent to validity of the unsubtracted dispersion relation. As mentioned in the Introduction, this crucial assumption is clearly spelled out as such in the original papers; in the following Sections of the paper we will investigate situations where the subtraction constant is present in the pion transition form factor.

The constant in the bound (8) may be given [33] in terms of the photon spectral density and the pion quark structure function, namely

$$
\left|F_{\pi^{0} \gamma \gamma^{*}}\left(Q^{2}\right)\right|<\frac{2 \sqrt{\Pi(\infty)}}{Q} \int_{0}^{1} d x \sqrt{\frac{F_{1}\left(x, Q^{2}\right)}{x(1-x)}},
$$

where

$$
\Pi(s)=\frac{s}{16 \pi^{3} \alpha_{\mathrm{QED}}^{2}} \sigma_{e^{+} e^{-} \rightarrow \operatorname{hadrons}}(s),
$$

with the asymptotic value

$$
\Pi(\infty)=\frac{1}{12 \pi^{2}} \sum_{i} e_{i}^{2}
$$

$e_{i}$ denoting the charge of the quark of flavor $i$ in the natural units. The pion structure function is

$$
F_{1}\left(x, Q^{2}\right)=\frac{1}{2} \sum_{i} e_{i}^{2}\left[q_{i}\left(x, Q^{2}\right)+\bar{q}_{i}\left(x, Q^{2}\right)\right]
$$


with $q_{i}\left(x, Q^{2}\right)$ and $\bar{q}_{i}\left(x, Q^{2}\right)$ denoting the quark and antiquark distribution functions at momentum $Q$.

With the help of the SMRS [59] and the GRV 60] parameterizations of the pion PDF's we obtain, via integrating from $x=10^{-5}$ to 1 , a numerical estimate for the coefficient in the bound (9) which actually exhibits a rather mild dependence on $Q^{2}$. With $Q^{2}$ in the range $10-40 \mathrm{GeV}^{2}$ we find for the LO and NLO evolved PDF's the bounds

$$
\begin{aligned}
\left|F_{\pi^{0} \gamma \gamma^{*}}\left(Q^{2}\right)\right| & <\frac{0.85(1)}{Q}(\mathrm{LO}) \\
& <\frac{0.75(1)}{Q}(\mathrm{NLO}),
\end{aligned}
$$

with the small uncertainty reflecting the considered $Q$ interval. Therefore the bound is completely "inefficient" in the considered range of momenta, as it is an order of magnitude above the BaBar data. For that reason it is of formal rather than practical interest. ${ }^{1}$

\section{BOUND FROM THE $Z \rightarrow \pi^{0} \gamma$ DECAY}

Another bound for the pion transition form factor comes from the rare $Z \rightarrow \pi^{0} \gamma$ decays. In that process, proceeding via a quark loop, only the vector coupling of the $Z$ boson to the quarks contributes [48], hence

$$
\frac{F_{Z \rightarrow \pi^{0} \gamma}\left(q^{2}\right)}{F_{Z \rightarrow \pi^{0} \gamma}(0)}=\frac{F_{\pi^{0} \gamma^{*} \gamma}\left(q^{2}\right)}{F_{\pi_{0} \gamma^{*} \gamma}(0)} .
$$

The experimental limit $\Gamma\left(Z \rightarrow \pi^{0} \gamma\right)<5 \times 10^{-5} \Gamma_{\text {tot }}(Z)=$ $10.25 \times 10^{-5} \mathrm{GeV}$, provided by the Particle Data Group [49], implies

$$
\left|F_{Z \rightarrow \pi^{0} \gamma}\left(M_{Z}^{2}\right) / F_{Z \rightarrow \pi^{0} \gamma}(0)\right|<0.17 .
$$

This experimental bound is not as stringent as predicted by many authors, where $\Gamma\left(Z \rightarrow \pi^{0} \gamma\right) \sim 10^{-11} \mathrm{GeV}$ (see, e.g., [50]). The bound can be used in models which predict the form factor in the time-like region, e.g. the Regge models considered in the following Sections.

\section{QUARK MODELS}

The role of the constituent vs current quarks in the $\pi^{0}$ and $\omega$ transition form factors in triangle diagrams was recognized in Ref. [61]. The diagram of Fig. 1] is superficially linearly divergent, but the requirement of the gauge invariance guarantees convergence. In the constituent

\footnotetext{
1 We are taking $Q^{2}$ large enough as to neglect the higher twist corrections. Actually the bound never crosses the pQCD limit $2 f_{\pi} / Q^{2}$ for the lowest momenta considered in Ref. [60], namely $Q_{0}^{2} \sim 0.26 \mathrm{GeV}^{2}$ at $\mathrm{LO}$ and $Q_{0}^{2} \sim 0.40 \mathrm{GeV}^{2}$ at NLO.
}

quark model (CQM) a direct computation of the triangle yields, for the general kinematic case,

$$
F_{\pi^{0} \gamma^{*} \gamma^{*}}\left(Q^{2}, A\right)=\frac{1}{4 \pi^{2} f_{\pi}} G\left(Q^{2}, A\right),
$$

where the loop function (we work in the strict chiral limit of $\left.m_{\pi}=0\right)$,

$$
\begin{aligned}
G\left(Q^{2}, A\right)= & \frac{2 M^{2}}{Q^{2}} \int_{0}^{1} \frac{d x}{x} \times \\
& \log \left[\frac{2 M^{2}+(1+A) x(1-x) Q^{2}}{2 M^{2}+(1-A) x(1-x) Q^{2}}\right],
\end{aligned}
$$

is normalized to unity at the origin, $G(0, A)=1$. For the special case $A=1$

$$
\begin{aligned}
& G\left(Q^{2}, A=1\right) \equiv G\left(Q^{2}\right)= \\
& \frac{2 M^{2}}{Q^{2}} \int_{0}^{1} \frac{d x}{x} \log \left[1+x(1-x) \frac{Q^{2}}{M^{2}}\right] .
\end{aligned}
$$

We note that the evaluation is covariant, thus different in philosophy from the conventional light-cone analysis. In the latter case the soft part of the diagram containing the PDA is factorized, while the hard part of the diagram is evaluated according to the light-cone Feynman rules. On the other hand, in CQM one evaluates the diagram of Fig. 1 with the instant-form Feynman rules, corresponding to local or non-local variants of the model. That way one is insensitive to the issues of factorization. However, it is not clear how credible this approach is at high virtualities, in particular in local models, where the virtual quark propagator carries, even asymptotically, a large constituent mass. In the study of this Section we are, however, primarily interested in formal aspects, namely, the TW bounds in a field-theoretic model, thus we shall not be concerned with the issue whether or not a lowenergy quark model can be realistically used to obtain the asymptotic transition form factor according to Fig. 1.

Chiral quark models are particular realizations of the large- $N_{c}$ limit. A variant of the CQM, the well-known Georgi-Manohar model [62], allows the quarks to carry an axial charge different from unity, $g_{A}^{Q} \neq 1$. The relevant part of the Lagrangian of the model is

$$
\begin{aligned}
& L=\bar{q}\left(i \not \partial+g_{A}^{Q} A \gamma_{5}-M\right) q+\frac{f^{2}}{4} \operatorname{Tr}\left(\partial_{\mu} U^{\dagger} \partial^{\mu} U\right)+\mathrm{WZW}, \\
& A_{\mu}=\frac{i}{2}\left(u^{\dagger} \partial_{\mu} u-u \partial_{\mu} u^{\dagger}\right), \quad u=e^{i \vec{\pi} \cdot \vec{\tau} /(2 f)}, \quad U=u^{2}, \quad
\end{aligned}
$$

where $f=93 \mathrm{MeV}$ is the pion decay constant and WZW denotes the Wess-Zumino-Witten term [63, 64]. There has been some discussion on whether or not $1-g_{A}^{Q}=$ $\mathcal{O}\left(N_{c}^{0}\right)$. This issue was answered in the affirmative way in Ref. [65], where the $\mathcal{O}\left(N_{c}^{0}\right)$ departure from unity is basically due to the $t$-channel exchanges in the AdlerWeisberger sum rule for the pion-quark scattering. In 
the NJL model with vector mesons, the $A_{1}-\pi$ mixing is an explicit leading- $N_{c}$ source [66 68] of the effect. Because of the chiral anomaly, the field representation used to describe the $g_{A}^{Q} \neq 1$ situation is relevant. In Ref. [69] it was shown that the apparent anomalous inequivalence of effective theories may be compensated by including extra terms in the effective action. More specifically, the original GM model does not contain the anomalous piece, such that the Wess-Zumino-Witten (WZW) action should be added a posteriori, as in Eq. (19). An example on how various vertex functions are modified by naively including $g_{A}^{Q} \neq 1$ and violating the anomaly in chiral quark models is presented in Ref. [70]. The subtraction procedure described above to restore the anomaly and based on [69] was addressed in Ref. [71] (see also [72]).

A direct calculation of the pion transition form factor in the GM model supplied with the WZW action yields the result

$$
F_{\pi^{0} \gamma \gamma^{*}}\left(Q^{2}\right)=\frac{1}{4 \pi^{2} f_{\pi}}+\frac{g_{A}^{Q}}{4 \pi^{2} f_{\pi}}\left[G\left(Q^{2}\right)-1\right],
$$

with $G\left(Q^{2}\right)$ given by Eq. (18). This expression satisfies the anomaly and the dispersion relation but does not vanish at infinity, since

$$
F_{\pi^{0} \gamma \gamma^{*}}\left(Q^{2}\right)=\frac{1-g_{A}^{Q}}{4 \pi^{2} f_{\pi}}+\frac{g_{A}^{Q} M^{2}}{4 \pi^{2} f_{\pi}} \frac{\left[\log \left(Q^{2} / M^{2}\right)\right]^{2}}{Q^{2}}+\ldots
$$

fulfills the first TW bound (for the imaginary part) but does not fulfill the second bound (for the real part).

Similarly, within SQM [20] a direct implementation of $g_{A}^{Q}$ is equally possible and one gets the result of Eq. (20) with

$$
G\left(Q^{2}\right)=\frac{1}{3}\left[\frac{2 m_{\rho}^{2}}{m_{\rho}^{2}+Q^{2}}+\frac{m_{\rho}^{2}}{Q^{2}} \log \left(\frac{m_{\rho}^{2}+Q^{2}}{m_{\rho}}\right)\right] .
$$

With $g_{A}^{Q}=1$ this model fulfills qualitatively the result of Radyushkin with a similar mass scale [17], since

$$
F_{\pi^{0} \gamma \gamma^{*}}\left(Q^{2}\right)=\frac{1-g_{A}^{Q}}{4 \pi^{2} f_{\pi}}+\frac{g_{A}^{Q} m_{\rho}^{2}}{12 \pi^{2} f_{\pi}} \frac{\left[\log \left(Q^{2} / m_{\rho}^{2}\right)\right]}{Q^{2}}+\ldots
$$

In the local quark models we have the general relation

$$
\begin{aligned}
F_{\pi^{0} \gamma \gamma^{*}}(t)= & \frac{1-g_{A}^{Q}}{4 \pi^{2} f_{\pi}}+\frac{g_{A}^{Q}}{12 \pi^{2} f}\left[2 F_{\pi^{+}}^{\mathrm{em}}(t)\right. \\
& \left.+\int_{0}^{t} d s F_{\pi^{+}}^{\mathrm{em}}(s)\right]
\end{aligned}
$$

which correlates the charge and transition form factors. This relation shows that in these models even in the case $g_{A}^{Q}-1$ one cannot have complete VMD simultaneously for the two form factors.
Unfortunately, attempts to fit the experimental data with the quark-model formulas, with $g_{A}$ equal or different from unity, are not numerically successful in the whole momentum range, unless one uses unrealistic model parameters. The purpose of the above calculations was different. Our examples show in an explicit manner that the second TW may be violated in models consistent with all field-theoretic constraints, such as covariance, gauge invariance, chiral symmetry, and anomaly matching. Importantly, they provide instances where the form factor does not vanish asymptotically. The calculation also illustrates that the $Q^{2}=0$ value and the finite $Q^{2}$ values are independent. The possibility of disobeying the second TW bound will be explored in the following Sections.

\section{VECTOR MESON DOMINANCE MODELS}

We now come to the core of our paper and consider several implementations of IVMD, both in a simple approach with a single vector meson, as well as in more sophisticated Regge models with infinitely-many radiallyexcited states. It will turn out that the pion transition form factor can be accurately described with this phenomenological method. The coupling of photons to vector mesons has a long history and Ref. [73] comprehensively reviews the interplay between universality, the vector-meson dominance and the low-energy theorems. We will highlight first the issue of IVMD for the charge form factor, such that our points are later on more easily made for the transition form factor.

\section{A. Charge form factor}

The charge form factor is the famous case where VMD can be implemented:

$$
F_{\pi^{+}}^{\mathrm{em}}(t)=\frac{M_{V}^{2}}{M_{V}^{2}-t} \rightarrow-\frac{M_{V}^{2}}{t}+\ldots
$$

with $t=-Q^{2}$. When the unregularized quark-loop mean squared radius [74] is matched to (25), one gets the relation

$$
M_{V}^{2}=24 \pi^{2} f_{\pi}^{2} / N_{c}
$$

There is no way to match the successful form factor of Eq. (25) to the pQCD result. ${ }^{2}$

\footnotetext{
2 If we nevertheless match Eq. 250 to pQCD, the only possible solution is $\alpha_{s}(Q)=\pi / 2$, which yields a too small scale $Q \sim$ $300 \mathrm{MeV}$. This matching to $\mathrm{pQCD}$ may seem weird but need not necessarily be conceptually wrong. Note that in the string model calculation of the $q \bar{q}$ potential one gets $V_{\bar{q} q}(r)=-\pi / 12 r$, whereas from pQCD $V_{\bar{q} q}(r)=-4 \alpha_{S} / 3 r$. This yields $\alpha=\pi / 16$, which means a safely high scale of $\mu=2 \mathrm{GeV}$ for $\Lambda_{Q C D}=$
} 
If one uses a once-subtracted dispersion relation and imposes the current conservation, $F_{\pi^{+}}^{\mathrm{em}}(0)=1$, one gets

$$
F_{\pi^{+}}^{\mathrm{em}}(t)-1=\frac{1}{\pi} \int_{t_{0}}^{\infty} \frac{t}{t^{\prime}} \frac{\operatorname{Im} F_{\pi^{+}}^{\mathrm{em}}\left(t^{\prime}\right)}{t^{\prime}-t-i \epsilon} d t^{\prime}
$$

which yields

$$
F_{\pi^{+}}^{\mathrm{em}}(-\infty)-1=-\frac{1}{\pi} \int_{t_{0}}^{\infty} \frac{\operatorname{Im} F_{\pi^{+}}^{\mathrm{em}}\left(t^{\prime}\right)}{t^{\prime}} d t^{\prime}
$$

Saturation with a single resonance gives the result

$$
F_{\pi^{+}}^{\mathrm{em}}(t)=1+\frac{a}{2} \frac{t}{M_{V}^{2}-t} \rightarrow 1-\frac{a}{2}+\frac{a}{2} \frac{M_{V}^{2}}{-t}+\ldots
$$

When the coupling $a / 2=f_{\rho \gamma} g_{\rho \pi \pi} / m_{\rho}^{2} \equiv g_{\rho \pi \pi} / g_{\rho e^{+} e^{-}}$ becomes unity, the form factor vanishes at infinity. Sakurai's universality indeed requires $g_{\rho \pi \pi}=g_{\rho e^{+} e^{-}}$. In general, however, we may have $g_{\rho \pi \pi} \neq g_{\rho e^{+} e^{-}}$. Note that positivity requires $a<2$, such that $F_{\pi^{+}}^{\mathrm{em}}\left(-Q^{2}\right)<1$. A fit to the experimental data $75-78]$ yields $a / 2=0.99(2)$ and $M_{V}=679(36) \mathrm{MeV}$. Thus the data for the pion charge form factor are consistent with the complete VMD, but certainly do not preclude IVMD, with $a / 2$ departing slightly from unity.

Caldi and Pagels [79] obtained a similar expression as Eq. (29) for the pion form factor from a direct photon contribution and a momentum dependent $\rho-\gamma$ vertex (see also Ref. [80]). The discussed properties are nicely displayed by the hidden-symmetry approach by Bando et al. [81], where there is a contact piece and the vector meson term, which dominates completely when the KSFR relation is fulfilled. Actually, in Ref. [82] the equivalence of this approach to more conventional ones is established. The interplay between VMD and universality was analyzed by Schechter [83] (see also Ref. [84]). Symmetry breaking effects have been analyzed in Ref. [85]. More attempts including predictions for meson decays can be found in $[86$.

Of course, the non vanishing of the low-energy representation (the one-resonance saturation) need not be taken as a fundamental problem. The only feature we see is that this non-vanishing represents more accurately the unknown high-energy data. If we had infinitely many states, we could fit that data and by separating explicitly the contribution from the lowest $\rho$-state. We see that the effect of all other states does behave as the $a$ constant, which is slowly dependent on $Q$ in a wide energy range.

Ideally, one should take the $e^{+} e^{-} \rightarrow \pi^{+} \pi^{-}$data over all possible momenta. However, obviously the experimental data are available only up to a certain maximum

$240 \mathrm{MeV}$. The string model describes accurately the lattice data in the short distance region. In the case of the charge form factor with VMD, the required scale is much smaller. value, $s_{\max }=4 \Lambda^{2}$. Thus, even if the form factor vanishes at infinity, we have

$$
\begin{aligned}
F_{\pi^{+}}^{\mathrm{em}}(t)-1= & \frac{1}{\pi} \int_{4 m_{\pi}^{2}}^{4 \Lambda^{2}} \frac{t}{s} \frac{\operatorname{Im} F_{\pi^{+}}^{\mathrm{em}}(s)}{s-t} d s \\
& +\frac{1}{\pi} \int_{4 \Lambda^{2}}^{\infty} \frac{t}{s} \frac{\operatorname{Im} F_{\pi^{+}}^{\mathrm{em}}(s)}{s-t} d s
\end{aligned}
$$

where the last term is weakly momentum-dependent and hence resembles a constant behavior assumed in IVMD, as discussed above.

\section{B. Transition form factor}

For the $\pi^{0} \gamma \gamma^{*}$ form factor the complete VMD with just one state implies

$$
F_{\pi^{0} \gamma \gamma^{*}}(t)=\frac{1}{4 \pi^{2} f} \frac{M_{V}^{2}}{M_{V}^{2}-t} \rightarrow-\frac{M_{V}^{2}}{4 \pi^{2} f t}+\ldots
$$

Note that while the anomaly value does not depend on $N_{c}$ explicitly, the high momentum behavior does. When matching Eq. (31) to the pQCD result of Eq. (5) is done, one gets independently the relation (26),

$$
M_{V}^{2}=24 \pi^{2} f_{\pi}^{2} / N_{c} .
$$

Despite this appealing property, the parametrization (31) fails to describe the experimental data in the high momentum region.

If we incorporate the possibility that the form factor need not vanish at infinity, we may write a oncesubtracted dispersion relation [87],

$$
F_{\pi^{0} \gamma \gamma^{*}}(t)-F_{\pi^{0} \gamma \gamma^{*}}(0)=\frac{1}{\pi} \int_{t_{0}}^{\infty} \frac{t}{t^{\prime}} \frac{\operatorname{Im} F_{\pi^{0} \gamma \gamma^{*}}\left(t^{\prime}\right)}{t^{\prime}-t-i \epsilon} d t^{\prime} .
$$

The influence of the well-known time-like region does not determine unambiguously when the onset of the pQCD takes place. Actually, the single VMD model shows that even in the space-like region with momenta as low as $Q^{2} \sim m_{\rho}^{2}$ the effects of the chiral logs and final state interactions are meager. Taking the limit $t \rightarrow-\infty$ we get

$$
F_{\pi^{0} \gamma \gamma^{*}}(-\infty)-F_{\pi^{0} \gamma \gamma^{*}}(0)=-\frac{1}{\pi} \int_{t_{0}}^{\infty} \frac{\operatorname{Im} F_{\pi^{0} \gamma \gamma^{*}}\left(t^{\prime}\right)}{t^{\prime}} d t^{\prime} .
$$

This shows that $F_{\pi^{0} \gamma \gamma^{*}}(-\infty)<F_{\pi^{0} \gamma \gamma^{*}}(0)$ if $\operatorname{Im} F_{\pi^{0} \gamma \gamma^{*}}(t)>0$. When we saturate the absorptive part with just one resonance, we get

$$
F_{\pi^{0} \gamma \gamma^{*}}\left(-Q^{2}\right)=\frac{1}{4 \pi^{2} f_{\pi}}\left[1-c \frac{Q^{2}}{M_{V}^{2}+Q^{2}}\right],
$$

with $M_{V}$ denoting the vector-meson mass. The coefficient $c$ is related to the $\rho \rightarrow \pi \gamma$ decay,

$$
c=c_{\rho \pi \gamma}=\frac{-2 e f_{\rho}}{m_{\rho}} \frac{\mathcal{A}\left(\rho^{0} \rightarrow \pi^{0} \gamma\right)}{\mathcal{A}\left(\pi^{0} \rightarrow \gamma \gamma\right)}=1.022 \pm 0.051,
$$




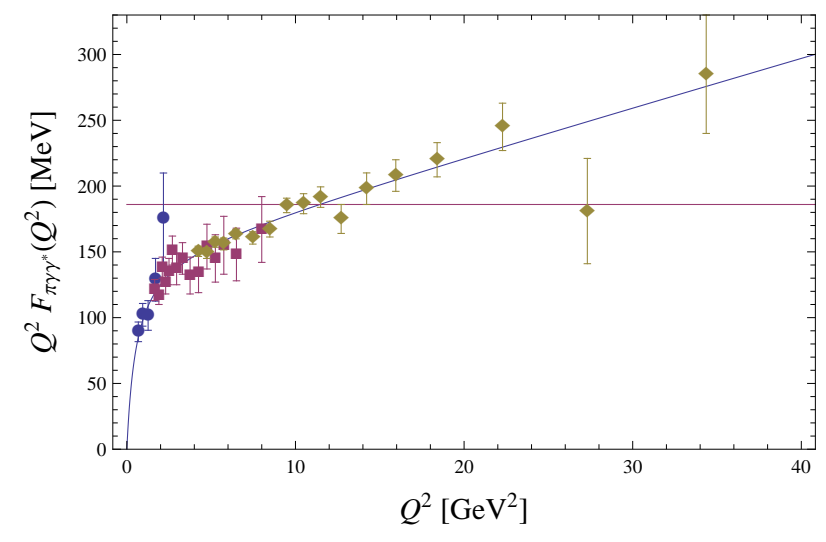

FIG. 2: The pion transition form factor in the space-like region $Q^{2}=-t>0$. The dots, squares, and diamonds correspond to the CELLO [88], CLEO 89], and BaBar 7] data, correspondingly. The line is the incomplete-vector-mesondominance fit with formula (35) and parameters (38).

where the value of the estimate is obtained from the decay width $\Gamma\left(\rho^{+} \rightarrow \pi^{+} \gamma\right)=68 \pm 7 \mathrm{KeV}$.

If we fit the parameters in formula (35) with the CLEO data only, we get

$$
c=0.998(18), \quad M_{V}=777(44) \mathrm{MeV},
$$

with $\chi^{2} / \mathrm{DOF}=0.54$, hence $c$ is consistent with unity, in agreement with previous determinations assuming complete VMD, however not excluding IVMD. On the other hand, the fit to the combined CELLO, CLEO, and BaBar data yields the result

$$
c=0.986(2), \quad M_{V}=748(14) \mathrm{MeV},
$$

hence $c$ is different form unity at the level of 7 standard deviations, while it remains consistent with estimate (36). As seen from Fig. 2, the agreement with the experimental data over all the momentum range is remarkable, with $\chi^{2} / \mathrm{DOF}=0.7$. The contours of the confidencelevels are displayed in Fig. 3 .

The logarithmic slope at the origin reads

$$
b_{\pi}=-\left.\left[\frac{1}{F_{\pi^{0} \gamma \gamma^{*}}(Q)} \frac{d}{d Q^{2}} F_{\pi^{0} \gamma \gamma^{*}}(Q)\right]\right|_{Q^{2}=0} .
$$

Numerically, taking the optimum parameters (38), we get the value

$$
b_{\pi}=\frac{c}{M_{V}^{2}}=1.76(7) \mathrm{GeV}^{-2} .
$$

Our IVMD model estimate is in a very good agreement with the average experimental values quoted in the PDG [49]: $b_{\pi}=(1.76 \pm 0.22) \mathrm{GeV}^{-2}$. This value is very close to that originally reported by the CELLO collaboration [88], obtained from an extrapolation from high- $Q^{2}$ data to low $Q^{2}$ by means of generalized vector meson dominance, $b_{\pi}=(1.4 \pm 1.3 \pm 2.6) \mathrm{GeV}^{-2}$ given in [90], and $b_{\pi}=(1.4 \pm 0.8 \pm 1.4) \mathrm{GeV}^{-2}$ given in [91].

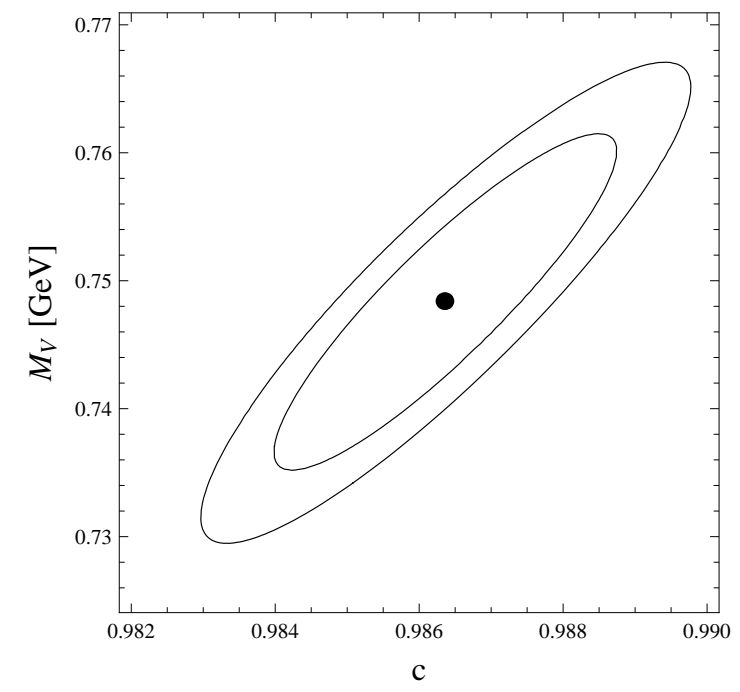

FIG. 3: The $\Delta \chi^{2}=2.3$ and 4.6 contours, corresponding to the $68 \%$ and $90 \%$ confidence levels, in the $M_{V}-c$ plane for the fit with the IVMD ansatz (35). The central values yield $\chi^{2} / \mathrm{DOF}=0.6$.

To end this Section, we turn to the rare decay $Z \rightarrow$ $\pi^{0} \gamma$, which probes the transition form factor in the physical region. From Eq. (35) with parameters (38) we get $\left|F_{Z \rightarrow \pi^{0} \gamma}\left(M_{Z}^{2}\right) / F_{Z \rightarrow \pi^{0} \gamma}(0)\right|=0.014(2)$, a comfortable order of magnitude smaller than the upper experimental bound (15), but not as small as predicted by several models ( $\sim 10^{-4}$, see, e.g., [50] and references therein).

\section{REGGE MODELS}

In the previous Section we have considered the simplest possible implementation of IVMD, with just one vector-meson state. However, the large- $N_{c}$ limit of QCD involves tree-level diagrams with infinitely many states, including the radial excitations. That way the matching to the QCD correlators can be accomplished [36, 42, 47], as well as the correct asymptotic behavior of the pion charge form factor [46] may be obtained. In this Section we analyze the pion transition form factors in the framework of Regge models with infinitely many radially excited vector-meson states.

Based on the success of the Veneziano-LovelaceShapiro dual resonance model (see, e.g., [92, 93] and references therein) Suura 94] and Frampton [95] proposed analytic models. The general form reads

$$
\begin{aligned}
F_{\pi^{0} \gamma^{*} \gamma^{*}}\left(Q^{2}, A\right) & =\sum_{V_{\rho}, V_{\omega}} \frac{F_{V_{\rho}}\left(q_{1}^{2}\right) F_{V_{\omega}}\left(q_{2}^{2}\right) G_{\pi V_{\rho} V_{\omega}}\left(q_{1}^{2}, q_{2}^{2}\right)}{\left(q_{1}^{2}-M_{V_{\rho}}^{2}\right)\left(q_{2}^{2}-M_{V_{\omega}}^{2}\right)} \\
& +\left(q_{1} \longleftrightarrow q_{2}\right),
\end{aligned}
$$

where $F_{V_{\rho}}$ and $F_{V_{\omega}}$ are the current-vector meson couplings, while $G_{\pi V_{\rho} V_{\omega}}$ is the coupling of two vector mesons to the pion. The situation is depicted in Fig. (4) At the 


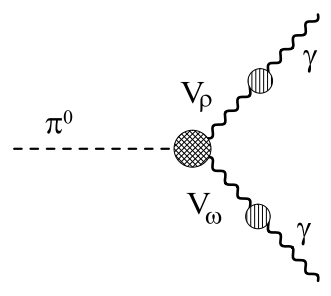

FIG. 4: The pion transition form factor in Regge models. The labels $V_{\rho}$ and $V_{\omega}$ denote the infinite vector meson towers with the $\rho$ and $\omega$ quantum numbers.

soft photon point, corresponding to the neutral pion decay $\pi^{0} \rightarrow 2 \gamma$, the chiral anomaly matching condition imposes the normalization

$$
\begin{aligned}
F_{\pi^{0} \gamma^{*} \gamma^{*}}(0,0) & =\sum_{V_{\rho} V_{\omega}} \frac{2 F_{V_{\rho}}(0) F_{V_{\omega}}(0) G_{\pi V_{\rho} V_{\omega}}(0,0)}{M_{V_{\rho}}^{2} M_{V_{\omega}}^{2}} \\
& =\frac{1}{4 \pi^{2} f} .
\end{aligned}
$$

This consistency constraint, realized in nature, can be always satisfied in models by an appropriate choice of the couplings. In Ref. [45] we analyzed the transition form factor with the help of formula (41) with the coupling constants $F_{V}$ taken as constants (as requested asymptotically by the matching of spectral densities to QCD) and allowing for constant diagonal couplings in $G_{\pi V_{\rho} V_{\omega}}$. As a result, a constant PDA of Eq. 6 was extracted. The corresponding pion transition form factor has the asymptotic behavior $\sim \log \left(Q^{2} / \mu^{2}\right) / Q^{2}$, however with physical values of the model parameters it overshoots the data in the region above $Q^{2} \sim 1 \mathrm{GeV}^{2}$, thus does not properly reproduce the data. ${ }^{3}$

In the analysis below we use a more sophisticated approach, namely the factorizable product suggested by Dominguez [96] in the dual description of radiative decays,

$$
F_{\pi^{0} \gamma \gamma^{*}}(t)=\frac{1}{4 \pi^{2} f_{\pi}} f_{b}(t)
$$

where

$$
\begin{aligned}
f_{b}(t) & =\frac{1}{B\left(b-1, \frac{M_{V}^{2}}{a}\right)} \\
& \times \sum_{n=0}^{\infty} \frac{\Gamma(2-b+n)}{\Gamma(n+1) \Gamma(2-b)} \frac{1}{a n+M_{V}^{2}-t} .
\end{aligned}
$$

The function $f_{b}(t)$ depends on three parameters: the lowest-lying vector-meson mass, $M_{V}$, the string tension,

\footnotetext{
${ }^{3}$ As already mentioned, we stress that taking the $Q^{2} \rightarrow \infty$ limit is an operational way of extracting the leading twist PDA; this is different than describing the current data within this limit.
}

$\sigma=a /(2 \pi)$, and the asymptotic fall-off parameter, $b$. The mass formula is then $M_{V}(n)^{2}=a n+M_{V}^{2}$. The function (45) fulfills the normalization condition

$$
f_{b}(0)=1 \text {. }
$$

For $x \gg y$ one has $B(x, y) \sim \Gamma(y) x^{-y}$, hence in the asymptotic region of $M_{V}^{2}-t \gg(b-1) a$ we find

$$
f_{b}(t) \sim \frac{\Gamma\left(\frac{M_{V}^{2}}{a}+b-1\right)}{\Gamma\left(\frac{M_{V}^{2}}{a}\right)}\left(\frac{a}{M_{V}^{2}-t}\right)^{b-1} .
$$

The TW bounds are satisfied if $b>1.5$.

We remark that in Ref. [46] this version of the Regge approach was used to describe the charge form factor. We have shown that it can be accurately reproduced up to $Q^{2} \sim 6 \mathrm{GeV}^{2}$, while the pQCD result greatly undershoots the experiment. The onset of pQCD occurs at extremely high ("cosmological") values of $Q^{2}$.

We now proceed with the application of the presented Regge model to the pion transition form factor. Taking $a=1.3 \mathrm{GeV}^{2}$ (which correspond to the string tension $\left.\sigma=(455 \mathrm{MeV})^{2}\right)$, a $\chi^{2}$ fit of formula (43) to the joint CELLO, CLEO, and BaBar data yields

$$
M_{V}=0.672(25) \mathrm{GeV}, \quad b=1.81(3),
$$

with $\chi^{2} / \mathrm{DOF}=1$. The fit is shown in Fig. 5] with the dashed line. Since $b>1.5$, the fit satisfies the TW bounds. For the logarithmic slope at the origin we find $b_{\pi}=2.1(2) \mathrm{GeV}^{-2}$. We also get, with smoothing the spectral density in the physical region, the ratio $\left|F_{Z \rightarrow \pi^{0} \gamma}\left(M_{Z}^{2}\right) / F_{Z \rightarrow \pi^{0} \gamma}(0)\right|=0.0014(4)$, which is comfortably below the experimental bound and an order of magnitude smaller than the IVMD fit of Sect. VIB.

We can further try to improve the agreement with the BaBar data by explicitly separating the first pole as follows:

$$
F_{\pi^{0} \gamma \gamma^{*}}(t)=\frac{1}{4 \pi^{2} f_{\pi}}\left[c_{\rho} \frac{m_{\rho}^{2}}{m_{\rho}^{2}-t}+\left(1-c_{\rho}\right) \frac{\bar{f}_{b}(t)}{\bar{f}_{b}(0)}\right],
$$

where $\bar{f}_{b}(t)$ is obtained from Eq. (45) with the $n=0$ term omitted from the sum. We impose the TW bound by setting $b=1.5$. Then (with $a=1.3 \mathrm{GeV}^{2}$ ) we find

$$
c=0.95(2), M_{V}=709(9) \mathrm{MeV}^{2},
$$

with $\chi^{2} / \mathrm{DOF}=0.85, b_{\pi}=1.9(1) \mathrm{GeV}^{-2}$, and $\left|F_{Z \rightarrow \pi^{0} \gamma}\left(M_{Z}^{2}\right) / F_{Z \rightarrow \pi^{0} \gamma}(0)\right|=0.01(1)$. The corresponding curve is presented in Fig. 5 with the dotted line.

Finally, we take a subtracted Regge model, in analogy to the model of Sec. VIB of the form

$$
F_{\pi^{0} \gamma \gamma^{*}}(t)=\frac{1}{4 \pi^{2} f_{\pi}}\left[1-c+c f_{b}(t)\right] .
$$

Setting $M_{V}=770 \mathrm{MeV}$ and $a=1.3 \mathrm{GeV}^{2}$ yields the optimum values for the remaining parameters

$$
c=0.984(4), \quad b=2.05(3),
$$




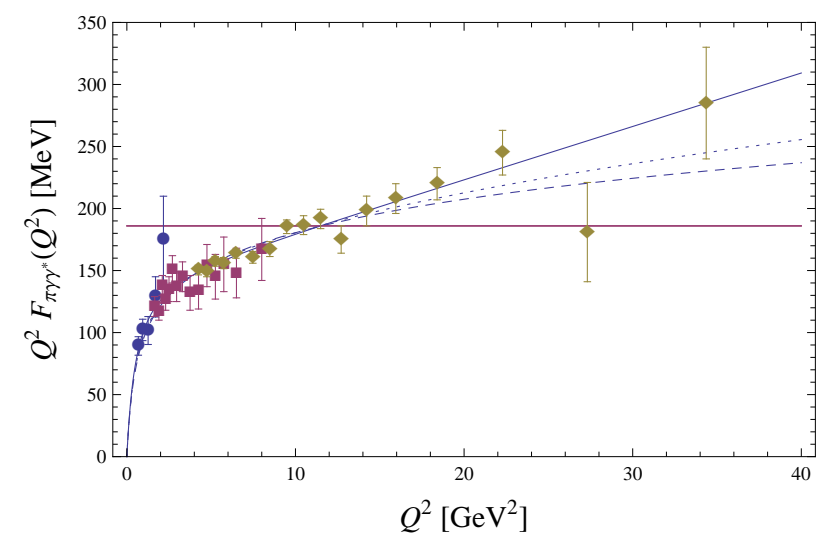

FIG. 5: The pion transition form factor in the space-like region $Q^{2}=-t>0$. The dots, squares, and diamonds correspond to the CELLO [88], CLEO [89], and BaBar 7] data, correspondingly. The dashed line is the results of the Regge fit with formula (43) and parameters (47). The dotted line shows the Regge fit with Eq.(48) and parameters (49). Finally, the solid line corresponds to the subtracted Regge model of Eq. (50) and parameters (51).

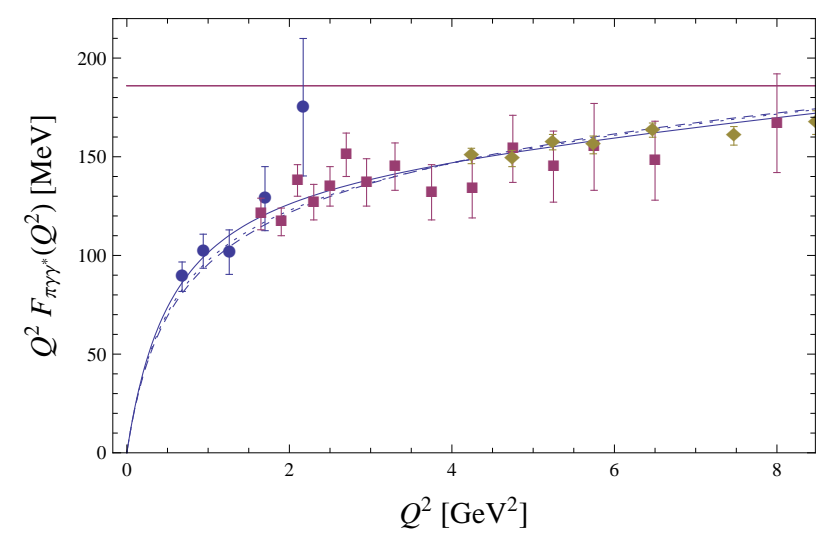

FIG. 6: Same as Fig. 5 for a smaller range of $Q^{2}$.

with $\chi^{2} / \mathrm{DOF}=0.7$. Since the value of $b$ is consistent with 2 , the model gives very similar results to the model with the single vector-meson state considered in Sec. VIB Hence we find $b_{\pi}=1.69(1) \mathrm{GeV}^{-2}$, and $\left|F_{Z \rightarrow \pi^{0} \gamma}\left(M_{Z}^{2}\right) / F_{Z \rightarrow \pi^{0} \gamma}(0)\right|=0.021(4)$. The corresponding curve is presented in Fig. 5 with the solid line. Within the present model we see that the higher radially excited states of the vector mesons, $\rho^{\prime}, \rho^{\prime \prime}, \ldots$ and $\omega^{\prime}, \omega^{\prime \prime}, \ldots$, are weakly coupled.

We also zoom the low- $Q^{2}$ range in Fig. 6. All the considered fits practically overlap in the displayed range, in agreement with the observation that IVMD could not be rejected even at $Q^{2} \sim 8 \mathrm{GeV}^{2}$. Our analysis shows that higher energy data do in fact favor IVMD at the level of 7 standard deviations.

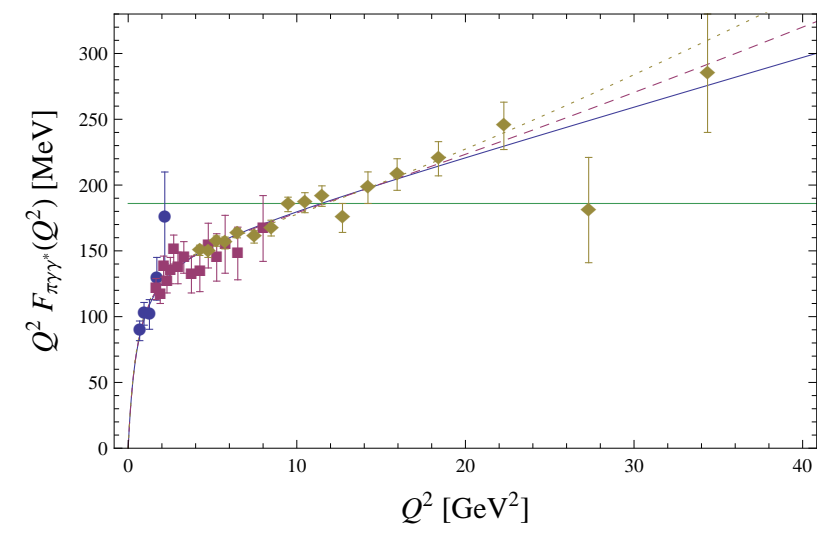

FIG. 7: The IMVD fit of Eq. (53) with $A=1,0.975$, and 0.95, denoted with solid, dashed, and dotted lines, respectively.

\section{ASYMMETRY PARAMETER}

As mention in the Introduction, in the BaBar kinematic setup $-q_{1}^{2}<0.6 \mathrm{GeV}^{2}$ and $-q_{2}^{2}>3 \mathrm{GeV}^{2}$, suggesting $A \sim 0.9-0.97$, hence $A$ is not strictly 1 . This departure has significance for the fits and the obtained parameters, which should not be forgotten in precision analyses. We take as an example the IMVD model, which now becomes

$$
\begin{aligned}
& F_{\pi^{0} \gamma \gamma^{*}}\left(-Q^{2}\right)=\frac{1}{4 \pi^{2} f_{\pi}} \times \\
& {\left[1-c\left(1-\frac{4 M_{V}^{4}}{4 M_{V}^{4}+4 M_{V}^{2} Q^{2}+\left(1-A^{2}\right) Q^{4}}\right)\right] .}
\end{aligned}
$$

Fitting with $A=1,0.975$, and 0.95 yields, respectively, $c=0.986,0.978,0.974$, and $M_{V}=748,754,768 \mathrm{MeV}$. We note significantly different values of the optimum parameters, with $M_{V}$ closest to the physical value for $A=0.95$. The corresponding curves are displayed in Fig. 7.

\section{CONCLUSIONS}

These are our main findings:

- The Terazawa-West bound, asserted for the real part of the pion transition form factor, need not be fulfilled in field-theoretic approaches. We provide an explicit counter-example with the low-energy Georgi-Manohar model, where the chiral anomaly is fulfilled but the transition form factor does not vanish at $t \rightarrow-\infty$. Provided this feature holds in QCD, it opens a possibility of explaining the BaBar experimental data with models incorporating the incomplete vector-meson dominance.

- Moreover, the coefficient in the Terazawa-West bound, estimated with the help of a phenomenological parametrization of the pion parton distribution 
functions, is large, extending an order of magnitude above the BaBar data. Thus, even if it holds in the absence of polynomial contributions to the real part, it is completely ineffective for the momentum range of interest.

- Already the simplest model with the incomplete vector-meson dominance, incorporating a single vector-meson state, is capable of reproducing the data in the whole available experimental range, $0<Q^{2}<35 \mathrm{GeV}^{2}$.

- Within the Regge approach, where infinitely many radially excited states are included, the data can be fitted both ways: satisfying or violating the TW bound. The agreement with the experiment is satisfactory, both near $Q^{2}-0$, where the anomaly value and the slope of the form factor are reproduced, as well as in the intermediate CLEO range and the high- $Q^{2}$ BaBar range.

- An additional constraint on the models in the timelike region of momenta follows from the rare $Z \rightarrow$ $\pi^{0} \gamma$ decay. We use this bound in our considerations. For the considered models it is comfortably satisfied.

- Finally, we note that the numerical fits are quite sensitive to the photon momentum asymmetry parameter, $A$, which leads to sensitivity in the physical parameters, such as the vector meson mass, and sensitivity of the transition form factor in the asymptotic range. Since $A$ is not strictly 1 , the effect of the kinematic cuts should be considered in precision analyses.
[1] S. L. Adler, Phys. Rev. 177, 2426 (1969).

[2] J. S. Bell and R. Jackiw, Nuovo Cim. A60, 47 (1969).

[3] G. P. Lepage and S. J. Brodsky, Phys. Lett. B87, 359 (1979).

[4] A. V. Efremov and A. V. Radyushkin, Phys. Lett. B94, 245 (1980).

[5] N. Isgur and C. H. Llewellyn Smith, Phys. Rev. Lett. 52, 1080 (1984).

[6] N. Isgur and C. H. Llewellyn Smith, Nucl. Phys. B317, 526 (1989).

[7] B. Aubert (The BABAR) (2009), 0905.4778.

[8] G. P. Lepage and S. J. Brodsky, Phys. Rev. D22, 2157 (1980).

[9] S. J. Brodsky and G. P. Lepage, Phys. Rev. D24, 1808 (1981).

[10] F. del Aguila and M. K. Chase, Nucl. Phys. B193, 517 (1981).

[11] E. Braaten, Phys. Rev. D28, 524 (1983).

[12] E. P. Kadantseva, S. V. Mikhailov, and A. V. Radyushkin, Yad. Fiz. 44, 507 (1986).

[13] A. P. Bakulev, S. V. Mikhailov, and N. G. Stefanis, Phys. Lett. B508, 279 (2001), hep-ph/0103119.

[14] A. P. Bakulev, S. V. Mikhailov, and N. G. Stefanis, Phys. Rev. D67, 074012 (2003), hep-ph/0212250.

[15] A. P. Bakulev, S. V. Mikhailov, and N. G. Stefanis, Phys. Lett. B578, 91 (2004), hep-ph/0303039.

[16] S. V. Mikhailov and N. G. Stefanis, Nucl. Phys. B821, 291 (2009), 0905.4004.

[17] A. V. Radyushkin, Phys. Rev. D80, 094009 (2009), 0906.0323

[18] E. Ruiz Arriola and W. Broniowski, Phys. Rev. D66, 094016 (2002), hep-ph/0207266.

[19] M. V. Polyakov, JETP Lett. 90, 228 (2009), 0906.0538.

[20] E. Ruiz Arriola and W. Broniowski, Phys. Rev. D67, 074021 (2003), hep-ph/0301202.

[21] S. V. Mikhailov and N. G. Stefanis, Mod. Phys. Lett. A24, 2858 (2009), 0910.3498.

[22] A. E. Dorokhov (2009), 0905.4577.

[23] A. E. Dorokhov (2009), 0909.5111.
[24] A. E. Dorokhov (2009), 0912.5278.

[25] S. Noguera and V. Vento (2010), 1001.3075.

[26] P. Kotko and M. Praszalowicz, Phys. Rev. D80, 074002 (2009), 0907.4044.

[27] N. I. Kochelev and V. Vento, Phys. Rev. D81, 034009 (2010), 0912.2172.

[28] A. E. Dorokhov (2010), 1003.4693.

[29] M. Knecht and A. Nyffeler, Eur. Phys. J. C21, 659 (2001), hep-ph/0106034.

[30] A. Nyffeler (2009), 0912.1441.

[31] H. Terazawa, Phys. Rev. D6, 2530 (1972).

[32] G. B. West, Phys. Rev. Lett. 30, 1271 (1973).

[33] H. Terazawa, Rev. Mod. Phys. 45, 615 (1973).

[34] G. B. West, Mod. Phys. Lett. A5, 2281 (1990).

[35] G. 't Hooft, Nucl. Phys. B72, 461 (1974).

[36] E. R. Arriola and W. Broniowski, Eur. Phys. J. A31, 739 (2007), hep-ph/0609266.

[37] M. Golterman and S. Peris, JHEP 01, 028 (2001), hepph/0101098.

[38] S. R. Beane, Phys. Rev. D64, 116010 (2001), hepph/0106022.

[39] S. R. Beane, Phys. Lett. B521, 47 (2001), hep$\mathrm{ph} / 0108025$.

[40] Y. A. Simonov, Phys. Atom. Nucl. 65, 135 (2002), hep$\mathrm{ph} / 0109081$.

[41] M. Golterman and S. Peris, Phys. Rev. D67, 096001 (2003), hep-ph/0207060.

[42] E. Ruiz Arriola and W. Broniowski, Phys. Rev. D73, 097502 (2006), hep-ph/0603263.

[43] S. S. Afonin, Phys. Lett. B576, 122 (2003), hepph/0309337.

[44] S. S. Afonin, Nucl. Phys. B779, 13 (2007), hep$\mathrm{ph} / 0606291$.

[45] E. Ruiz Arriola and W. Broniowski, Phys. Rev. D74, 034008 (2006), hep-ph/0605318.

[46] E. Ruiz Arriola and W. Broniowski, Phys. Rev. D78, 034031 (2008), 0807.3488.

[47] E. R. Arriola and W. Broniowski (2010), 1001.1636.

[48] M. Jacob and T. T. Wu, Phys. Lett. B232, 529 (1989). 
[49] C. Amsler et al. (Particle Data Group), Phys. Lett. B667, 1 (2008).

[50] M. Bando and M. Harada, Prog. Theor. Phys. 92, 583 (1994), hep-ph/9307365.

[51] A. Schmedding and O. I. Yakovlev, Phys. Rev. D62, 116002 (2000), hep-ph/9905392.

[52] A. P. Bakulev, S. V. Mikhailov, and N. G. Stefanis, Phys. Rev. D73, 056002 (2006), hep-ph/0512119.

[53] S. S. Agaev, Phys. Rev. D72, 114010 (2005), hep$\mathrm{ph} / 0511192$.

[54] E. M. Aitala et al. (E791), Phys. Rev. Lett. 86, 4768 (2001), hep-ex/0010043.

[55] A. P. Bakulev, S. V. Mikhailov, and N. G. Stefanis, Annalen Phys. 13, 629 (2004), hep-ph/0410138.

[56] W. Broniowski and E. R. Arriola (2009), 0910.0869.

[57] E. Ruiz Arriola, Acta Phys. Polon. B33, 4443 (2002), hep-ph/0210007.

[58] W. Broniowski, E. R. Arriola, and K. Golec-Biernat, Phys. Rev. D77, 034023 (2008), 0712.1012.

[59] P. J. Sutton, A. D. Martin, R. G. Roberts, and W. J. Stirling, Phys. Rev. D45, 2349 (1992).

[60] M. Gluck, E. Reya, and I. Schienbein, Eur. Phys. J. C10, 313 (1999), hep-ph/9903288.

[61] M. Bando and M. Harada, Phys. Rev. D49, 6096 (1994), hep-ph/9307366.

[62] A. Manohar and H. Georgi, Nucl. Phys. B234, 189 (1984).

[63] J. Wess and B. Zumino, Phys. Lett. B37, 95 (1971).

64] E. Witten, Nucl. Phys. B223, 433 (1983).

[65] W. Broniowski, A. Steiner, and M. Lutz, Phys. Rev. Lett. 71, 1787 (1993), hep-ph/9304292.

[66] S. Klimt, M. Lutz, U. Vogl, and W. Weise, Nucl. Phys. A516, 429 (1990).

[67] U. Vogl, M. Lutz, S. Klimt, and W. Weise, Nucl. Phys. A516, 469 (1990).

[68] E. Ruiz Arriola, Phys. Lett. B253, 430 (1991).

[69] A. Manohar and G. W. Moore, Nucl. Phys. B243, 55 (1984).

[70] E. Ruiz Arriola and L. L. Salcedo, Phys. Lett. B316, 148 (1993).

[71] J. Bijnens and J. Prades, Phys. Lett. B320, 130 (1994), hep-ph/9310355.

[72] E. Ruiz Arriola and L. L. Salcedo, Nucl. Phys. A590, 703 (1995), nucl-th/9501026.

[73] H. B. O'Connell, B. C. Pearce, A. W. Thomas, and A. G. Williams, Prog. Part. Nucl. Phys. 39, 201 (1997), hep$\mathrm{ph} / 9501251$.

[74] R. Tarrach, Z. Phys. C2, 221 (1979).

[75] C. J. Bebek et al., Phys. Rev. D17, 1693 (1978).

[76] J. Volmer et al. (The Jefferson Lab F(pi)), Phys. Rev. Lett. 86, 1713 (2001), nucl-ex/0010009.

[77] T. Horn et al. (Jefferson Lab F(pi)-2), Phys. Rev. Lett. 97, 192001 (2006), nucl-ex/0607005.

[78] V. Tadevosyan et al. (Jefferson Lab F(pi)), Phys. Rev. C75, 055205 (2007), nucl-ex/0607007.

[79] D. G. Caldi and H. Pagels, Phys. Rev. D15, 2668 (1977).

[80] H. B. O'Connell, B. C. Pearce, A. W. Thomas, and A. G. Williams, Phys. Lett. B354, 14 (1995), hep-ph/9503332.

[81] M. Bando, T. Kugo, S. Uehara, K. Yamawaki, and T. Yanagida, Phys. Rev. Lett. 54, 1215 (1985).

[82] M. Benayoun, H. B. O'Connell, and A. G. Williams, Phys. Rev. D59, 074020 (1999), hep-ph/9807537.

[83] J. Schechter, Phys. Rev. D34, 868 (1986).

[84] A. R. Zerwekh, Acta Phys. Polon. B38, 2077 (2007), hep-ph/0603096.

[85] M. Harada and J. Schechter, Phys. Rev. D54, 3394 (1996), hep-ph/9506473.

[86] F. Klingl, N. Kaiser, and W. Weise, Z. Phys. A356, 193 (1996), hep-ph/9607431.

[87] T. N. Truong (2001), hep-ph/0102300.

[88] H. J. Behrend et al. (CELLO), Z. Phys. C49, 401 (1991).

[89] J. Gronberg et al. (CLEO), Phys. Rev. D57, 33 (1998), hep-ex/9707031.

[90] F. Farzanpay et al., Phys. Lett. B278, 413 (1992).

[91] R. Meijer Drees et al. (SINDRUM-I), Phys. Rev. D45, 1439 (1992).

[92] G. Veneziano, Phys. Rept. 9, 199 (1974).

[93] S. Mandelstam, Phys. Rept. 13, 259 (1974).

[94] H. Suura, Phys. Rev. Lett. 23, 551 (1969).

[95] P. H. Frampton, Phys. Rev. D1, 3141 (1970).

[96] C. A. Dominguez, Phys. Rev. D28, 2314 (1983). 